\title{
Trastuzumab Deruxtecan: A Quantum Leap in HER2-Positive Breast Cancer
}

\begin{abstract}
Docetaxel, trastuzumab, and pertuzumab, known as THP, is the preferred first-line treatment for HER2-positive advanced breast cancer, and the second-line drug of choice is trastuzumab emtansine. Most patients eventually develop resistance to systemic therapy. Trastuzumab deruxtecan, a novel HER2-targeted antibody drug conjugate, has shown to be promising in this subset. It is a HER2-targeted antibody drug conjugate structurally composed of humanized anti-HER2 monoclonal antibody, cleavable tetra-peptide-based liker, and a potent payload (topoisomerase 1 inhibitor: Exatecan). A phase 2 trial of heavily pretreated advanced HER2-positive breast cancer (median of six lines of prior therapy) showed an overall response of $61 \%$ and a median progression-free survival of 16 months. In December 2019, the Food and Drug Administration announced accelerated approval of trastuzumab deruxtecan for HER2-positive advanced breast cancer patients who were prior exposed to two or more lines of anti-HER2 therapy in a metastatic setting.
\end{abstract}

Keywords: Advanced breast cancer, HER2 positive, trastuzumab deruxtecan

\section{Introduction}

Docetaxel, trastuzumab, and pertuzumab, known as THP, is the preferred first-line treatment for HER2-positive advanced breast cancer and has shown a median overall survival (OS) of $4 \frac{1}{2}$ years. ${ }^{[1]}$ The second-line drug of choice is trastuzumab emtansine (TDM1). ${ }^{[2]}$ Resistance to anti-HER2 therapy develops due to various factors including loss of HER2 expression, downregulation of HER2 expression, heterogeneous HER2 expression, and receptor mutation. ${ }^{[3]}$

There is no standard third-line therapy for patients who progress after exposure to TDM1. Recently, tucatinib ${ }^{[4]}$ in combination with trastuzumab and capecitabine has shown to be promising in this subset, especially in those with brain metastasis. Trastuzumab deruxtecan, a novel HER2-targeted antibody drug conjugate, has shown to be promising in patients with heavily pretreated HER2-positive advanced breast cancer.

\section{Mechanism of Action}

Trastuzumab deruxtecan is a HER2-targeted antibody drug conjugate structurally

This is an open access journal, and articles are distributed under the terms of the Creative Commons Attribution-NonCommercial-ShareAlike 4.0 License, which allows others to remix, tweak, and build upon the work non-commercially, as long as appropriate credit is given and the new creations are licensed under the identical terms.

For reprints contact: reprints@medknow.com composed of humanized anti-HER2 monoclonal antibody, cleavable tetra-peptide-based liker, and a potent payload (topoisomerase 1 inhibitor: Exatecan). ${ }^{[5]}$ The monoclonal antibody targets HER2-expressing tumor cells and internalizes the payload. The lysosomes cleave the linker, causing the payload to inhibit topoisomerase 1, and cause tumor cell death.

\section{Landmark Trials}

\section{Preclinical}

Trastuzumab deruxtecan (DS-8201a) significantly suppressed tumor growth in immunocompetent mouse models with human HER2-expressing cell lines. It enhanced antitumor immunity by increased expression of dendritic cell markers, augmenting the expression of major histocompatibility complex Class I in tumor cells, and rejection of rechallenged tumor cells by adaptive immune cells. ${ }^{[6]}$

\section{Phase 1}

This dose-expansion study ${ }^{[7]}$ included 115 patients with heavily pretreated (seven prior lines) HER2-positive advanced breast cancer. The overall response rate was

How to cite this article: Dhanushkodi $M$ Trastuzumab deruxtecan: A quantum leap in HER2-positive breast cancer. Indian J Med Paediatr Oncol 2019;40:556-8.

\section{Manikandan Dhanushkodi}

Department of Medical Oncology, Cancer Institute (WIA), Chennai, Tamil Nadu, India

Submitted: 23-Dec-2019 Revised: 03-Jan-2020 Accepted: 06-Jan-2020 Published: 17-Feb-2020

Address for correspondence: Dr. Manikandan Dhanushkodi, Department of Medical Oncology, Cancer Institute (WIA), 38 Sardar Patel Road, Chennai - 600 036, Tamil Nadu, India.

E-mail:dmani1982@gmail.com

Access this article online

Website: www.ijmpo.org

DOI: 10.4103/ijmpo.ijmpo_264_19 Quick Response Code:
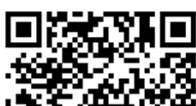

12ty

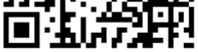


$60 \%$, the median time to response was 1.5 months, and the median progression-free survival (PFS) and OS were 22 months and not reached, respectively. The recommended phase 2 dose was $5.4 \mathrm{mg} / \mathrm{kg}$ or $6.4 \mathrm{mg} / \mathrm{kg} .{ }^{[8]}$

\section{Phase 2}

The Destiny-Breast01 trial ${ }^{[9]}$ included 184 patients with a median age of 55 years, 38\% of Asian ethnicity with a median tumor size of $5.5 \mathrm{~cm}$. This cohort included a heavily pretreated subset with a median of six lines of prior therapy (range: 2-27). All patients were prior exposed to trastuzumab and TDM1 and $66 \%$ had received pertuzumab. The overall response rate was $61 \%$, with a median PFS of 16 months.

\section{Phase 3}

The Destiny-Breast02 trial will assess the efficacy and safety of trastuzumab deruxtecan versus investigators' choice in patients who progress on TDM1. The Destiny-Breast03 trial will assess the efficacy and safety of trastuzumab deruxtecan versus TDM1.

\section{Advantages}

The remarkable response of trastuzumab deruxtecan is due to the highly potent payload (topoisomerase 1 inhibitor: Exatecan), high drug-to-antibody ratio ( 8 with trastuzumab deruxtecan and 3.5 with TDM1), stable linker payload in circulation, tumor-selective cleavable linker, and payload-induced bystander effect. ${ }^{[10]}$

\section{Novelty}

Trastuzumab deruxtecan has also shown activity in patients with low HER2-expressing (immunohistochemistry $<3+$ and negative in situ hybridization) ${ }^{[11]}$ breast cancer.

\section{Approval}

\section{Breakthrough therapy}

In August 2017, the Food and Drug Administration (FDA) granted breakthrough therapy designation to trastuzumab deruxtecan for the treatment of patients with advanced HER2-positive breast cancer previously treated with trastuzumab and pertuzumab and whose disease progressed after TDM1.

\section{Accelerated approval}

In December 2019, the FDA granted accelerated approval for trastuzumab deruxtecan for the treatment of adults with unresectable or metastatic HER2-positive breast cancer who had received two or more lines of anti-HER2-based regimen in a metastatic setting.

\section{Dose}

The recommended dose is $5.4 \mathrm{mg} / \mathrm{kg}$ every 3 weeks until disease progression/unacceptable toxicity.

\section{Side Effects}

The grade 3 or 4 adverse effects are neutropenia $(20 \%)$, anemia (9\%), and nausea ( $8 \%$ ). The potential serious adverse effect is interstitial lung disease (ILD) (Grade 1-2: 11\%; Grade 3-4: 0.5\%; and Grade 5: 2\%).

\section{Monitoring}

Patients need to be monitored closely for fever, cough, or dyspnea for early detection of ILD. Patients who develop ILD should be managed with steroids, dose reductions, or discontinuation.

\section{Other HER2-Positive Cancers}

Trastuzumab deruxtecan is also being evaluated in HER2-positive gastro-esophageal cancer, gastric cancer, colon cancer, and HER2 mutated non-small cell lung cancer.

\section{Newer Anti-HER2 Drugs in Pipeline}

- Tucatinib in combination with trastuzumab and capecitabine has shown a survival advantage in pretreated HER2-positive breast cancer, especially those with brain metastasis ${ }^{[4]}$

- Neratinib in combination with capecitabine has shown improved PFS as compared to lapatinib with capecitabine in pretreated HER2-positive advanced breast cancer ${ }^{[12]}$

- Margetuximab and chemotherapy improves PFS as compared to trastuzumab and chemotherapy in pretreated HER2-positive advanced breast cancer. ${ }^{[13]}$

\section{Conclusion}

Trastuzumab deruxtecan is a novel antibody drug conjugate with impressive and durable response in heavily pretreated HER2-positive advanced breast cancer.

\section{Financial support and sponsorship}

Nil.

\section{Conflicts of interest}

There are no conflicts of interest.

\section{References}

1. Swain SM, Baselga J, Kim SB, Ro J, Semiglazov V, Campone M, et al. Pertuzumab, trastuzumab, and docetaxel in HER2-positive metastatic breast cancer. $N$ Engl J Med 2015;372:724-34.

2. Verma S, Miles D, Gianni L, Krop IE, Welslau M, Baselga J, et al. Trastuzumab emtansine for HER2-positive advanced breast cancer. N Engl J Med 2012;367:1783-91.

3. Pohlmann PR, Mayer IA, Mernaugh R. Resistance to trastuzumab in breast cancer. Clin Cancer Res 2009;15:7479-91.

4. Murthy RK, Loi S, Okines A, Paplomata E, Hamilton E, Hurvitz SA, et al. Tucatinib, Trastuzumab, and Capecitabine for HER2-Positive Metastatic Breast Cancer. N Engl J Med 2019. 
5. $\mathrm{Xu} \mathrm{Z}$, Guo $\mathrm{D}$, Jiang $\mathrm{Z}$, Tong $\mathrm{R}$, Jiang $\mathrm{P}$, Bai L, et al. Novel HER2-targeting antibody-drug conjugates of trastuzumab beyond T-DM1 in breast cancer: Trastuzumab deruxtecan (DS-8201a) and (Vic-) trastuzumab duocarmazine (SYD985). Eur J Med Chem 2019;183:111682.

6. Iwata TN, Ishii C, Ishida S, Ogitani Y, Wada T, Agatsuma T. A HER2-targeting antibody-drug conjugate, trastuzumab deruxtecan (DS-8201a), enhances antitumor immunity in a mouse model. Mol Cancer Ther 2018;17:1494-503.

7. Tamura K, Tsurutani J, Takahashi S, Iwata H, Krop IE, Redfern C, et al. Trastuzumab deruxtecan (DS-8201a) in patients with advanced HER2-positive breast cancer previously treated with trastuzumab emtansine: A dose-expansion, phase 1 study. Lancet Oncol 2019;20:816-26.

8. Doi T, Shitara K, Naito Y, Shimomura A, Fujiwara Y, Yonemori $\mathrm{K}$, et al. Safety, pharmacokinetics, and antitumour activity of trastuzumab deruxtecan (DS-8201), a HER2-targeting antibody-drug conjugate, in patients with advanced breast and gastric or gastro-oesophageal tumours: A phase 1 dose-escalation study. Lancet Oncol 2017;18:1512-22.

9. Modi S, Saura C, Yamashita T, Park YH, Kim SB, Tamura K, et al. Trastuzumab deruxtecan in previously treated HER2-positive breast cancer. N Engl J Med 2019.

10. Nakada T, Sugihara K, Jikoh T, Abe Y, Agatsuma T. The latest research and development into the antibody-drug conjugate, [fam-] trastuzumab deruxtecan (DS-8201a), for HER2 cancer therapy. Chem Pharm Bull (Tokyo) 2019;67:173-85.

11. Modi S, Tsurutani J, Tamura K, Park H, Sagara Y, Murthy R, et al. Abstract P6-17-02: Trastuzumab deruxtecan (DS-8201a) in subjects with HER2-low expressing breast cancer: Updated results of a large phase 1 study. Cancer Res 2019;79:P6-17-02.

12. Saura C, Oliveira M, Feng YH, Dai MS, Hurvitz SA, Kim SB. Neratinib + capecitabine versus lapatinib + capecitabine in patients with HER2+ metastatic breast cancer previously treated with $\geq 2$ HER2-directed regimens: Findings from the multinational, randomized, phase III NALA trial. J Clin Oncol 2019;37:1002.

13. Rugo HS, Im SA, Wright GL, Escriva-de-Romani S, DeLaurentiis M, Cortes J. SOPHIA primary analysis: A phase 3 (P3) study of margetuximab (M)+chemotherapy (C) versus trastuzumab $(\mathrm{T})+\mathrm{C}$ in patients (pts) with HER2 + metastatic (met) breast cancer (MBC) after prior anti-HER2 therapies (Tx). J Clin Oncol 2019;37. 